\title{
The charged top-pion production associated with the bottom quark pair as a probe of the topcolor-assisted technicolor model at the $\mathrm{LHC}$
}

\author{
Guo-Li Liu ${ }^{a, *}$, Ping Zhou ${ }^{a, b, \dagger}$ \\ a Department of Physics, ZhengZhou University, Zhengzhou, Henan, China \\ ${ }^{b}$ Institut für Strahlenphysik, Forschungszentrum \\ Dresden-Rossendorf, 01314 Dresden, Germany
}

\begin{abstract}
The topcolor-assisted technicolor (TC2) model predicts the existence of the charged top-pions $\left(\pi_{t}^{ \pm}\right)$, whose large couplings with the third generation fermions will induce the charged top-pion production associated with the bottom and anti-bottom quark pair at the CERN Large Hadron Collider (LHC) through the parton processes $c \bar{b} \rightarrow \pi_{t}^{+} b \bar{b}$ and $u \bar{d}(c \bar{s}) \rightarrow \pi_{t}^{+} b \bar{b}$. In this paper we examine these productions and find that, due to the small Standard Model backgrounds, their production rates can exceed the $3 \sigma$ sensitivity of the LHC in a large part of parameter space, so these processes may serve as a good probe of the TC2 model.
\end{abstract}

PACS numbers: 12.60.Nz, 13.85.Hd, 13.85.Lg

*Email address: guoliliu@zzu.edu.cn

${ }^{\dagger}$ Email address: pzhou@fzd.de 


\section{INTRODUCTION}

The mechanism of electroweak symmetry breaking (EWSB) remains the most prominent mystery in elementary particle physics. Probing EWSB will be one of the most important tasks in the high energy colliders. Dynamical EWSB, such as technicolor (TC) theory [1], is an attractive idea that avoids the shortcomings of triviality and unnaturalness arising from the elementary Higgs field.

Among various kinds of technicolor theories, the topcolor scenario [2] is attractive because it can explain the large top quark mass and provides a possible EWSB mechanism. TC2 model [3] is one of the phenomenologically viable model, which has all essential features of the topcolor scenario. This model predicts three CP-odd top-pions $\pi_{t}^{0}, \pi_{t}^{ \pm}$and one CP-even top-higgs $h_{t}^{0}$ with large couplings to the third family, which may make these new scalar particles have a distinct experimental signature[4]. Thus, discovery of the scalar particles in future high energy colliders would be a definite signal of new physics beyond the standard model (SM), which would help us to understand the scalar sector and more importantly what lies beyond the SM.

LHC has already started its operation, and it will have considerably capability to discover and measure almost all the quantum properties of a SM higgs boson of any mass [5]. However, from the theoretical view point, it would be expected that the SM is replaced by a more fundamental theory at the $\mathrm{TeV}$ scale. If hadron colliders find evidence for a new scalar state, it may not necessarily be the SM Higgs boson. Many alternative new physics theories, such as supersymmetry, technicolor and little Higgs, predict the existence of new scalars or pseudo-scalar particles. These new particles may have so large cross sections and branching fractions as to be observable at the high energy colliders. Thus, studying the production of the new scalars at the LHC will serve as a powerful tool of the new physics models.

In this paper, we study how the technicolor models affect the charged top-pion production associated with the bottom quark pair processes $c \bar{b} \rightarrow \pi_{t}^{+} b \bar{b}$ and $u \bar{d}(c \bar{s}) \rightarrow \pi_{t}^{+} b \bar{b}$ via the new couplings in the TC2 model. In Sec. II, the technicolor model relative to our calculations is briefly reviewed. Sec. III shows the the numerical results for the different processes, respectively and analysis simply the SM backgrounds and the detectable probability. Summary and discussions are given in Sec. IV. 


\section{ABOUT THE TC2 MODEL}

The TC2 model predicts a number of charged bosons like the top-pions at the weak scale[3]. These scalars have large Yukawa couplings to the quarks at tree-level, among which the top-bottom and the charm-bottom couplings to the charged top-pion $\pi_{t}^{ \pm}$are most significant. Such couplings will induce bottom anti-bottom pair productions associated with a charged scalar at the LHC through the parton processes $c \bar{b} \rightarrow \pi_{t}^{+} b \bar{b}$ and $u \bar{d}(c \bar{s}) \rightarrow \pi_{t}^{+} b \bar{b}$. In this paper we will examine these productions and figure out if their rates can exceed the $3 \sigma$ sensitivity of the LHC. Since in the SM, such signals of the productions have unobservably small backgrounds at the LHC, these processes will serve as a probe for the TC2 model if their TC2 predictions can be above the $3 \sigma$ sensitivity.

Before our calculations we recapitulate the basics of TC2 model. The TC2 model[3] combines technicolor interaction with topcolor interaction, with the former being responsible for electroweak symmetry breaking and the latter for generating large top quark mass. The top quark mass is generated from two sources: one is from the extended technicolor (proportional to $\epsilon$ ) and the other from the topcolor (proportional to $1-\epsilon$ ). So the mass matrix of up-type quarks is composed of both extended technicolor and topcolor contributions. The diagonalization of this mass matrix will induce flavor changing top quark interactions in the Yukawa couplings which involve the composite scalars from topcolor and technicolor condensations, respectively.

The relevant couplings with the top-pion and the fermions can be written as 6 ]

$$
\mathcal{L}_{Y}=\frac{(1-\epsilon) m_{t}}{\sqrt{2} F_{t}} \frac{\sqrt{v_{w}^{2}-F_{t}^{2}}}{v_{w}}\left(\sqrt{2} K_{U R}^{t t *} K_{D L}^{b b} \bar{t}_{R} b_{L} \pi_{t}^{-}+\sqrt{2} K_{U R}^{t c *} K_{D L}^{b b} \bar{c}_{R} b_{L} \pi_{t}^{-}\right)
$$

where $K_{D L}$ and $K_{U R}$ are the rotation matrices that transform the weak eigenstates of lefthanded down-type and right-handed up-type quarks to their mass eigenstates, respectively. According to the analysis of [6], their favored values are given by

$$
K_{D L}^{b b} \simeq 1, \quad K_{U R}^{t t} \simeq \frac{m_{t}^{\prime}}{m_{t}}=1-\epsilon, \quad K_{U R}^{t c} \leq \sqrt{1-\left(K_{U R}^{t t}\right)^{2}}=\sqrt{2 \epsilon-\epsilon^{2}},
$$

In Eq.(1) we neglected the mixing between up quark and top quark. The factor $\sqrt{v_{w}^{2}-F_{t}^{2}} / v_{w}\left(v_{w} \simeq 174 \mathrm{GeV}\right)$ reflects the effect of the mixing between the top-pions and the would-be Goldstone bosons [7]. 
The total hadronic cross section for $p p \rightarrow \pi_{t}^{+} b \bar{b}+X$ can be obtained by folding the subprocess cross section $\hat{\sigma}$ with the parton luminosity

$$
\sigma(s)=\int_{\tau_{0}}^{1} d \tau \frac{d L}{d \tau} \hat{\sigma}(\hat{s}=s \tau)
$$

where $\tau_{0}=\left(2 m_{b}+m_{\pi}\right)^{2} / s$, and $s$ is the $p p$ center-of-mass energy squared. $d L / d \tau$ is the parton luminosity given by

$$
\frac{d L}{d \tau}=\int_{\tau}^{1} \frac{d x}{x}\left[f_{p_{1}}^{p}(x, Q) f_{p_{2}}^{p}(\tau / x, Q)+\left(p_{1} \leftrightarrow p_{2}\right)\right],
$$

where $f_{p_{1}}^{p}$ and $f_{p_{2}}^{p}$ are the parton $p_{1}$ and $p_{2}$ distribution functions in a proton, respectively. For our case, they could be $u, d, c, s$ and $b$ quark.

In our numerical calculation, the hadronic cross section at the LHC is obtained by convoluting the parton cross section with the parton distribution functions. In our calculations we use CTEQ6L [8] to generate the parton distributions with the renormalization scale $\mu_{R}$ and the factorization scale $\mu_{F}$ chosen to be $\mu_{R}=\mu_{F}=2 m_{b}+m_{\pi}$.

\section{CALCULATIONS AND RESULTS}

At the LHC, the cross sections of the charged top-pion production comes mainly from the quark collision processes $c \bar{b}, u \bar{d}, c \bar{s} \rightarrow \pi_{t}^{+} b \bar{b}$, as shown in Fig,1. In our numerical calculation, we use FormCalc for the three phase space integration [9].

For the SM parameters, we will take $m_{u}=2 \mathrm{MeV}, m_{d}=3 \mathrm{MeV}, m_{s}=100 \mathrm{MeV}$, $m_{c}=1.27 \mathrm{GeV}, m_{b}=4.5 \mathrm{GeV}, m_{t}=172.0 \mathrm{GeV}, m_{Z}=91.2 \mathrm{GeV}, m_{W}=80.399 \mathrm{GeV}[10]$.

The TC2 parameters involved in our calculations are the masses of the top-pions, the parameter $K_{U R}^{t c}$, the top-pion decay constant $F_{t}$ and the parameter $\epsilon$ which parametrizes the portion of the extended-technicolor contribution to the top quark mass. The masses of the charged top-pion mass are constrained from the absence of $t \rightarrow \pi_{t}^{+} b$, which gives a lower bound of $165 \mathrm{GeV}$ [11], and also from $R_{b}$ data, which yields a lower bound of about $250 \mathrm{GeV}$ [12]. In our numerical results we will take $F_{t}=50 \mathrm{GeV}, \epsilon=0.1, K_{U L}^{t t}=1$, $K_{U R}^{t t}=0.9$ and retain $m_{\pi}$ and $K_{U R}^{t c}$ as free parameters with $200 \leq m_{\pi} \leq 600 \mathrm{GeV}$ and $K_{U R}^{t c} \leq \sqrt{2 \epsilon-\epsilon^{2}}=0.43$.

In the following we present the results for the hadronic production cross section $c \bar{b} \rightarrow \pi_{t}^{+} b \bar{b}$ and $u \bar{d}(c \bar{s}) \rightarrow \pi_{t}^{+} b \bar{b}$, respectively. 


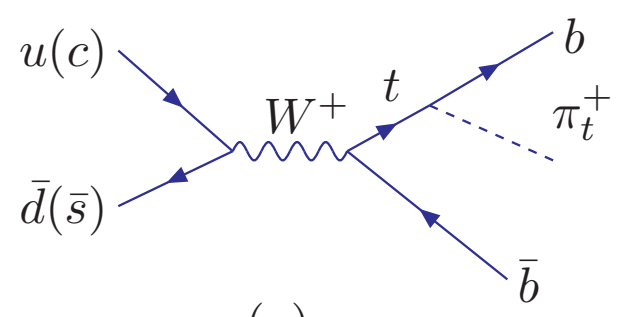

(a)
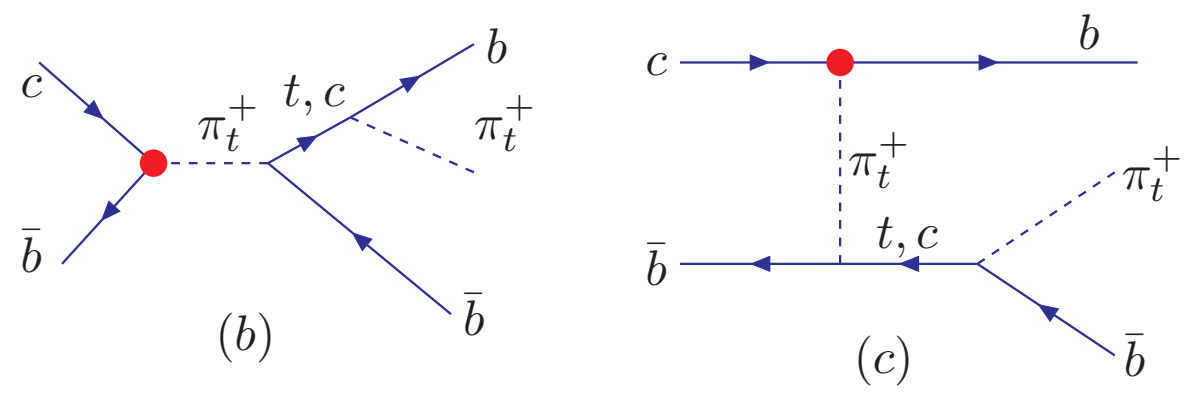

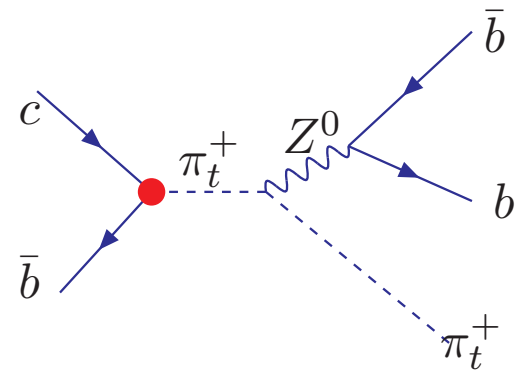

$(d)$

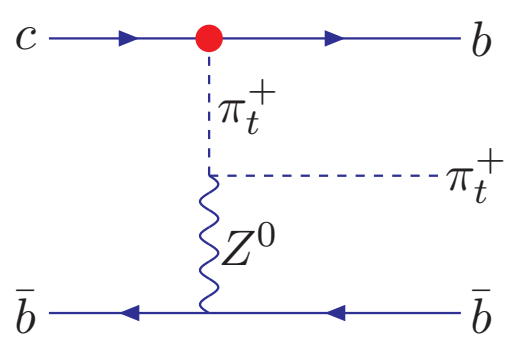

$(e)$

FIG. 1: Feynman diagrams for parton-level process $u \bar{d}(c \bar{s}) \rightarrow \pi_{t}^{+} b \bar{b}$ and $c \bar{b} \rightarrow \pi_{t}^{+} b \bar{b}$.

\section{A. The parton level process $c \bar{b} \rightarrow \pi_{t}^{+} b \bar{b}$}

The process is carried through out as the Fig!1(b)(c)(d)(e), containing one or more $\pi_{t}^{+} c \bar{b}$ vertexes, which is proportional to the TC2 parameter $K_{U R}^{t c}$.

Fig 2 shows that the hadronic cross section versus top-pion mass for different values of $K_{U R}^{t c}$. The cross section, which is about several hundreds fb in most of the parameter space, decreases with the increasing top-pion mass.

We see that the cross section increases with the increasing $K_{U R}^{t c}$ since the cross section is mainly proportional to $\left(K_{U R}^{t c}\right)^{2}$, with the vertex $\pi_{t}^{+} c \bar{b}, \sim K_{U R}^{t c}$, which can also be seen in Fig. 3 ,

Fig 3 gives the different contributions of the cross section from the exchange of top, charm quark and $Z^{0}$ gauge boson, which correspond to the different contribution including top quark, charm quark and the $Z^{0}$ propagator shown in Fig.1(b)(c)(d)(e). From which we 


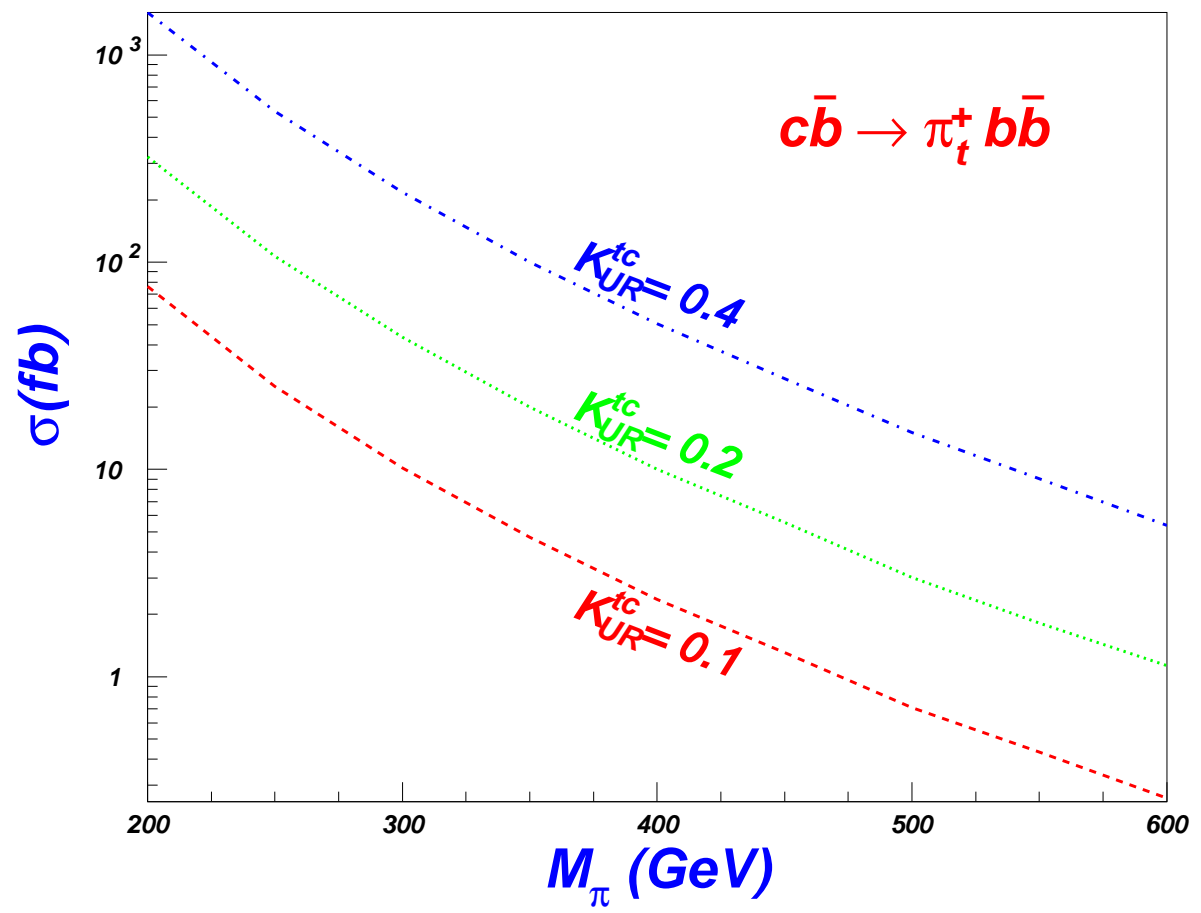

FIG. 2: Hadronic cross section for the production via $c \bar{b} \rightarrow \pi_{t}^{+} b \bar{b}$ at the LHC versus top-pion mass for $K_{U R}^{t c}=0.1,0.2,0.4$, respectively.
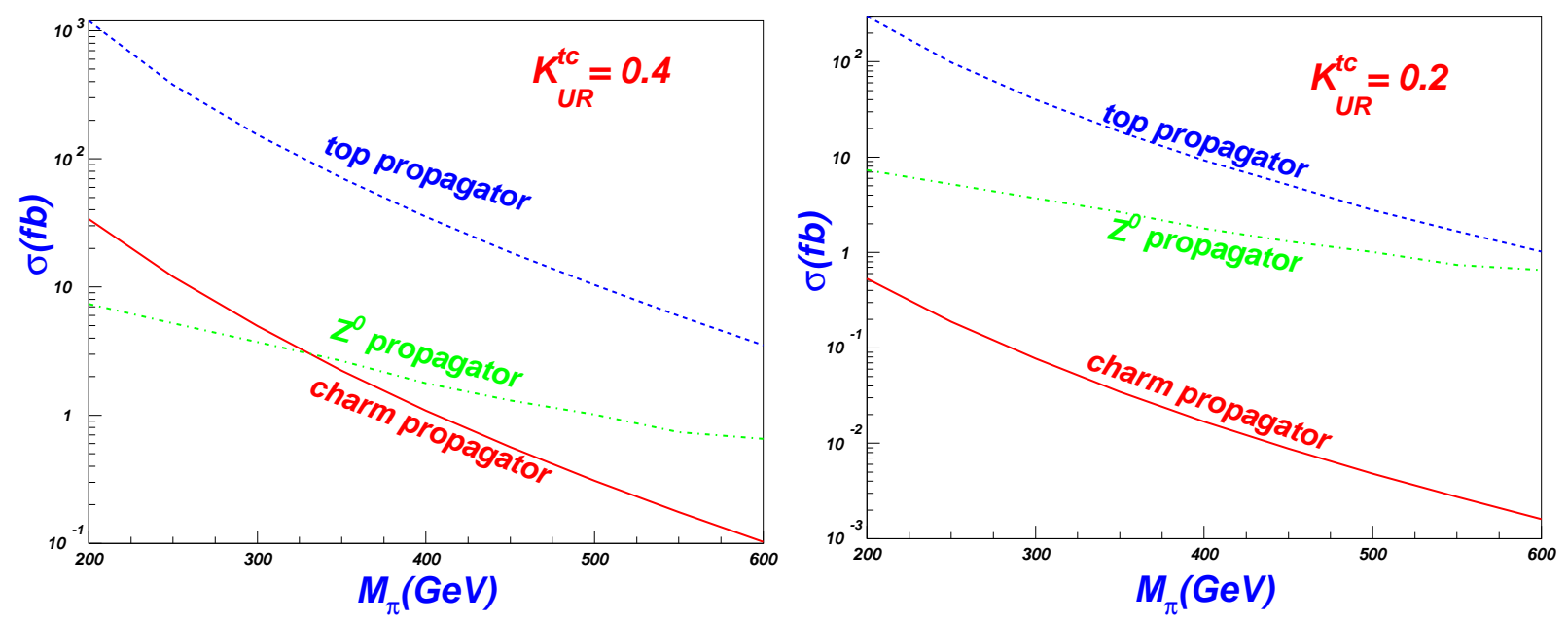

FIG. 3: Different contribution to $c \bar{b} \rightarrow \pi_{t}^{+} b \bar{b}$ at the LHC versus top-pion mass for $K_{U R}^{t c}=0.4$ and 0.2 , respectively.

can see that the top quark propagator contribution is the most largest since $\pi_{t}^{+} t \bar{b}$ couples largest. 


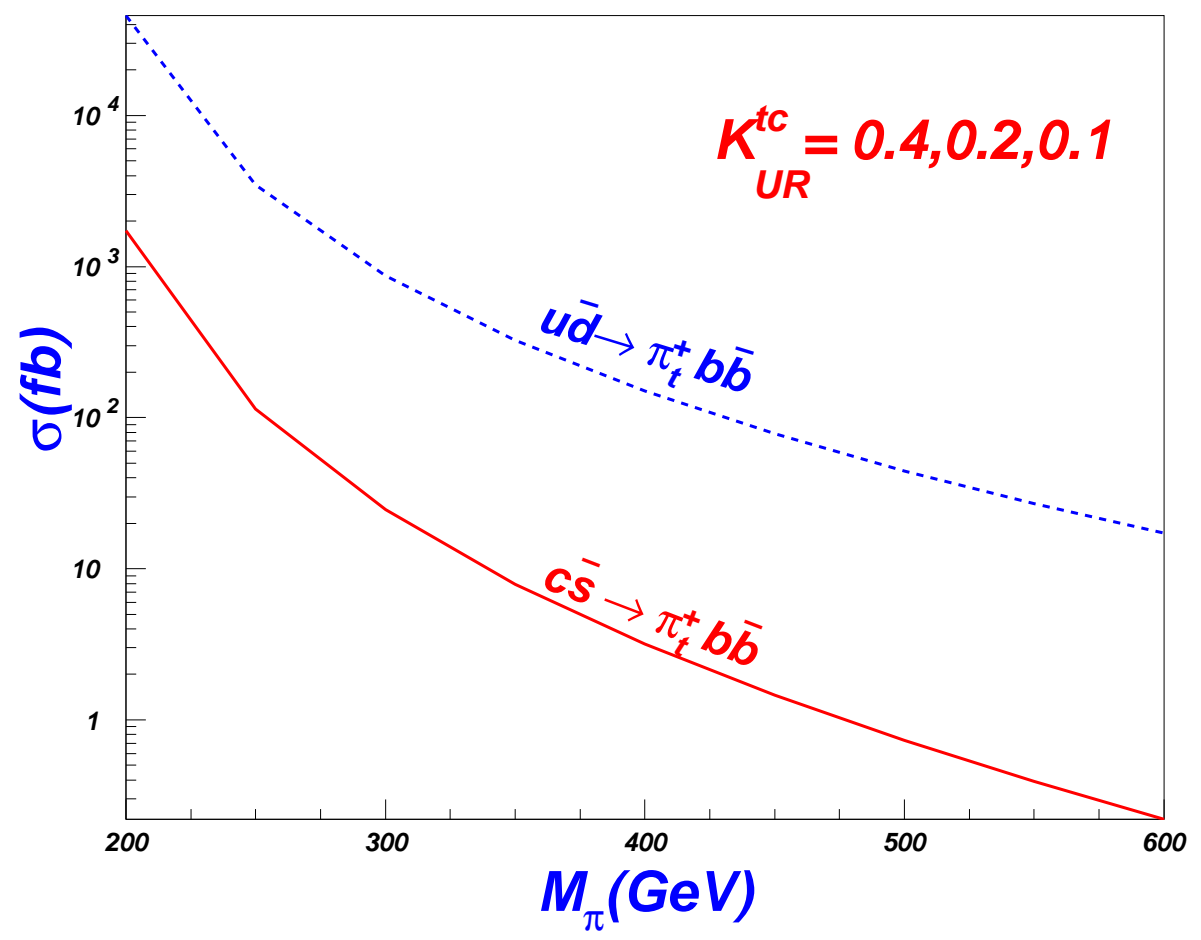

FIG. 4: Hadronic cross section for the production via $u \bar{d} \rightarrow \pi_{t}^{+} b \bar{b}$ and $c \bar{s} \rightarrow \pi_{t}^{+} b \bar{b}$ at the LHC versus top-pion mass.

\section{B. The processes $u \bar{d} \rightarrow \pi_{t}^{+} b \bar{b}$ and $c \bar{s} \rightarrow \pi_{t}^{+} b \bar{b}$}

Fig 4 shows the dependence of the cross sections of the two processes on the top-pion masses, from which we can see that the production rates is at the order of $1000 \mathrm{fb}$ in a large parameter space.

We can see from Fig 4 that the production rate of $u \bar{d} \rightarrow \pi_{t}^{+} b \bar{b}$ are larger than that of the $c \bar{s} \rightarrow \pi_{t}^{+} b \bar{b}$, which is easy to understand since what makes the difference is only the parton distribution functions when we neglect the light quark masses.

Comparing Fig, 2 and Fig, 4 , we can arrive at the conclusion that the cross sections of the $c \bar{b}$ collision is smaller than that of the $u \bar{d}$, which is also determined mainly by the parton distribution functions since the couplings in the two processes are almost in the same order of the two processes, i.e, the coupling $W^{+} u \bar{d} \sim e /\left(\sqrt{2} \sin \theta_{W}\right)$ is approximately equal to that of the $\pi_{t}^{+} c \bar{b} \sim m_{t} /\left(\sqrt{2} F_{t}\right) K_{U R}^{t c}$, where $\theta_{W}$ is the Weinberg angle. As to the process $c \bar{s} \rightarrow \pi_{t}^{+} b \bar{b}$, with the similar parton distribution functions as that of $c \bar{b} \rightarrow \pi_{t}^{+} b \bar{b}$, the cross sections of them are almost equivalent. 


\section{Observability of the processes}

For the productions of $P P \rightarrow \pi_{t}^{+} b \bar{b}+X$ we search for the final states from the subsequent decays $\pi_{t}^{+} \rightarrow c \bar{b}$, so the signal may be $3 b+j$. The main SM background at the LHC is the production of $Z Z, Z h$ and $h h$ (with the bottom quark miss-detected as charm quark). The signal will be picked out via the top-pion mass reconstruction since in the SM, the CKM element $V_{c b}$ is so small that they may not produce large signal, so we estimate the production rates arrive at $10 \mathrm{fb}$ may be detected by the LHC.

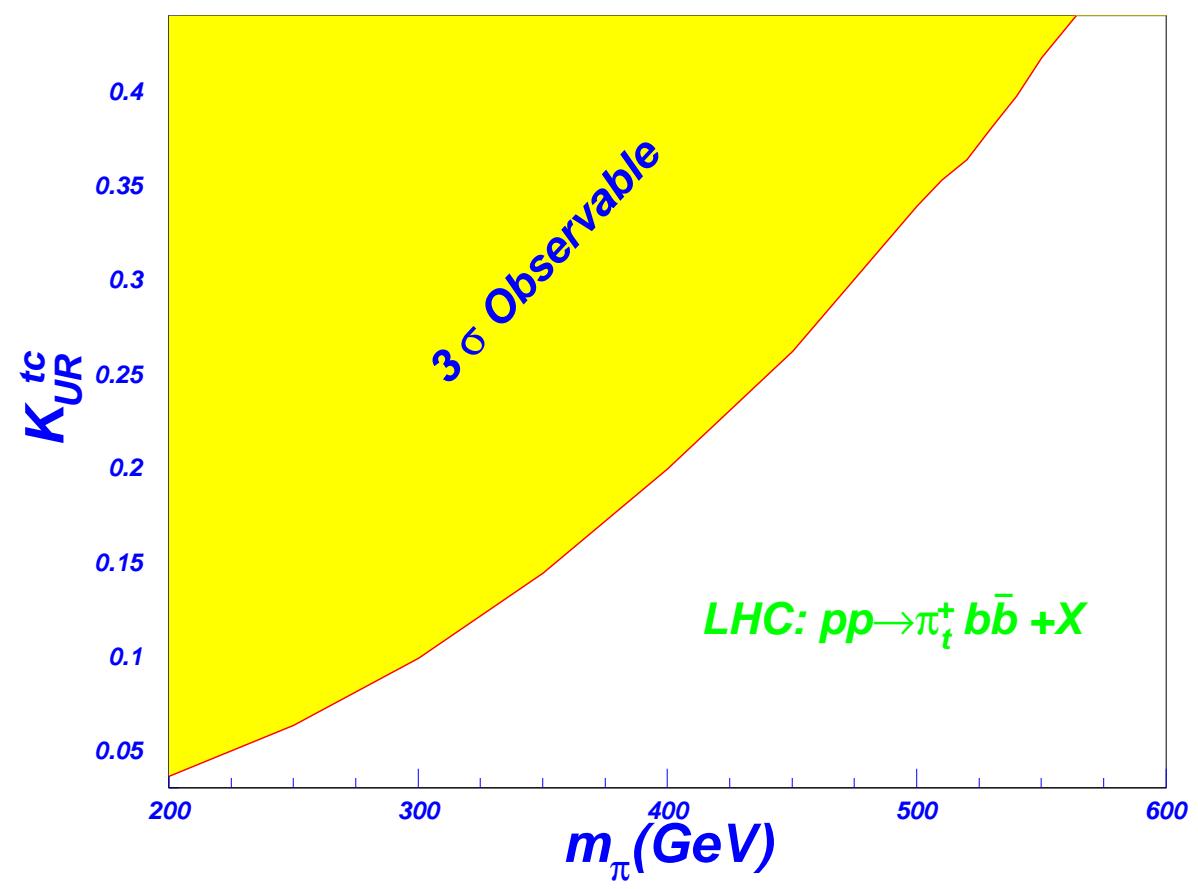

FIG. 5: The contour of $3 \sigma$ sensitivity (10 fb) for the cross section of the production via $c \bar{b} \rightarrow \pi_{t}^{+} b \bar{b}$ at the LHC in the plane of $K_{U R}^{t c}$ versus top-pion mass.

To show the observability of the production of $c \bar{b} \rightarrow \pi_{t}^{+} b \bar{b}+X$, we plot in Fig. 5 the contour of the cross section of the $3 \sigma$ sensitivity $(10 \mathrm{fb})$ in the plane of $K_{U R}^{t c}$ versus the top-pion mass. We see that in a large part of the parameter space the cross section can exceed the $3 \sigma$ sensitivity.

For the production $u \bar{d} \rightarrow \pi_{t}^{+} b \bar{b}$, the cross section is larger than $10 \mathrm{fb}$ in the full space of the the $m_{\pi}\left(200 \leq m_{\pi} \leq 600 \mathrm{Gev}\right)$. As for the production $c \bar{s} \rightarrow \pi_{t}^{+} b \bar{b}$, as long as the top-pion mass is smaller than $340 \mathrm{GeV}$, the cross sections will be larger than $10 \mathrm{fb}$ and may be detected at the LHC. 


\section{SUMMARY AND CONCLUSION}

In conclusion, we examined the charged top-pion productions associated with a bottom pair at the LHC in topcolor-assisted technicolor model. We found that their production rates can exceed the $3 \sigma$ sensitivity of the LHC in a large part of parameter space. Therefore, these processes will serve as a good probe for the topcolor-assisted technicolor model.

\section{Acknowledgments}

We would like to thank Junjie Cao and Jin Min Yang for helpful discussions.

[1] S. Weinberg, Phys. Rev. D13 (1976) 974; ibid, D19 (1979) 1277; L. Susskind, Phys. Rev. D20, (1979) 2619; E. Farhi, L. Susskind, Phys.Rept.74, (1981) 277.

[2] G. Cvetic, Rev. Mod. Phys. 71, 513 (1999); S. P. Martin, Phys. Rev. D46, 2197 (1992); Phys. Rev. D45, 4283 (1992), Nucl. Phys. B 398, 359 (1993); M. Lindner and D. Ross, Nucl. Phys. B 370, 30 (1992); R. Bonisch, Phys. Lett. B268, 394 (1991); C. T. Hill, D. C. Kennedy, T. Onogi, H. L. Yu, Phys. Rev. D 47, 2940 (1993).

[3] C. T. Hill, Phys. Lett. B 345, 483 (1995); K. Lane and E. Eichten, Phys. Lett. B 352, 383 (1995); K. Lane, Phys. Lett. B 483, 96 (1998); G. Cvetic,Rev. Mod. Phys.71, 513 (1999); C. T. Hill and E. H. Simmons, Phys. Rept. 381, 235 (2003); Erratum-ibid. 390 (2004) 553.

[4] See, e.g., G. Liu, et al., Phys. Rev. D 82, 115032 (2010); Chin. Phys. Lett26, 101401 (2009); Science China, 53, 1 (2010); Commun. Theor. Phys. 55, 852 (2011); Chin. Phys. C32, 697 (2008); arXiv: 1105.2607; J. Cao, et al., Phys. Rev. D 76, 014004 (2007); Eur. Phys. Jour. C 41, 381 (2005); Phys. Rev. D 70,114035 (2004); H. J. Zhang, Phys. Rev. D 77, 057501 (2008); X. L. Wang et al., Phys. Rev. D 50, 5781 (1994); C. Yue, et al., Phys. Lett. B 496, 93 (2000);

[5] ATLAS Collaboration, Technical Design Report, CERN-LHCC-99-15; CMS Collaboration, Technical Proposal, CERN-LHCC-94-38; G. Weiglein, et al., [LHC/LC Study Group], hep-ph/0410364.

[6] See, e.g., H. J. He and C. P. Yuan, Phys. Rev. Lett. 83, 28 (1999); C. Balazs, H.-J. He, C.P. 
Yuan, Phys.Rev. D60 (1999) 114001; H.-J. He, S. Kanemura, C. P. Yuan, Phys. Rev. Lett. 89, 101803 (2002); Phys. Rev. D 68, 075010 (2003). G. Burdman, Phys. Rev. Lett. 83, 2888 (1999); J. Cao, Z. Xiong, J. M. Yang, Phys. Rev. D 67, 071701 (2003); F. Larios and F.

Penunuri, J. Phys. G30, 895 (2004).

[7] G. Burdman, D. Kominis, Phys. Lett. B 403, 107 (1997).

[8] J. Pumplin, et al., JHEP 0602, 032 (2006).

[9] http://www.feynarts.de/formcalc/.

[10] K. Nakamura et al. (Particle Data Group), J. Phys. G37, 075021 (2010).

[11] B. Balaji, Phys. Lett. B 393, 89 (1997).

[12] G. Burdman and D. Kominis, Phys. Lett. B 403, 101 (1997); W. Loinaz and T. Takeuchi, Phys. Rev. D 60, 015005 (1999); C. Yue, Y. P. Kuang, X. L. Wang and W. Li, Phys. Rev. D 62, 055005 (2000). 